\title{
GRB 060206: Evidence of Precession of Central Engine
}

\author{
X.W. Liu*, X.F. Wu ${ }^{* \dagger}$ and T. Lu* \\ ${ }^{*}$ Purple Mountain Observatory, Chinese Academy of Science, Nanjing 210008, China \\ $\uparrow$ Theoretical Astrophysics 130-33, California Institute of Technology, Pasadena, California 91125
}

\begin{abstract}
The high-redshift ( $z=4.048)$ gamma-ray burst GRB 060206 showed unusual behavior, with a significant re-brightening about $3000 \mathrm{~s}$ after the burst. We assume that the central engine became active again $2000 \mathrm{~s}$ after the main burst and drove another more collimated off-axis jet. The two jets both interacted with the ambient medium and contributed to the whole emission. We numerically fit this optical afterglow from the two jets using the forward-shock model and the forward-reverse shock model. Combining with the zero time effect, we suggest that the fast rise at $\sim 3000 \mathrm{~s}$ in the afterglow was due to the off-axis emission from the second jet. The precession of the torus or accretion disk of the gamma ray burst engine is the natural explanation for the symmetry axes of these two jets not to lie on the same line.
\end{abstract}

Keywords: gamma-ray:bursts; jets

PACS: $98.70 . \mathrm{Rz}$; 98.38.Fs

\section{INTRODUCTION}

The gamma-ray burst (GRB) 060206 at Galactic Coordinates $1=78.07 \mathrm{deg}, \mathrm{b}=78.28 \mathrm{deg}$ triggered Swift-BAT on Feb. 6, 04:46:53 UT (trigger time $t=0$ ). It showed a single peak, with a duration of $T_{90}=7 \pm 2 \mathrm{~s}$ and the total fluence of $8.4 \pm 0.4 \times 10^{-7} \mathrm{erg} / \mathrm{cm}^{2}$ in the $15-350 \mathrm{keV}$ band [1]. The redshift was spectroscopically measured to be $z=4.048$ [2] Using the best fit low energy photon index $\Gamma_{1}=1.06 \pm 0.34$ and peak energy $E_{\text {peak }}$ of $75.4 \pm 19.5 \mathrm{keV}$ with the Band model with a fixed high energy photon index $\Gamma_{2}=2.5$, the isotropic-equivalent energy integrated from 1 to $10000 \mathrm{keV}$ in the burster's rest frame is $E_{\gamma, \text { iso }}=5.8 \times 10^{52} \mathrm{erg}$ [1].

This burst showed five evident bumps in the R-band light curve. An empirical model to fit this optical afterglow with the sum of a number of smoothly connected broken power-laws was adopted by Monfardini et al. (2006).

$$
F_{v}(t)=\sum_{j} F_{j}\left[\frac{2}{\left(t / t_{j}\right)^{-\alpha_{1, j} \cdot n}+\left(t / t_{j}\right)^{-\alpha_{2, j} \cdot n}}\right]^{1 / n}
$$

The temporal index $\alpha_{2}$ of each post-bump segment follows the normal decay slope $3(p-1) / 4$ predicted by the standard fireball model except the big-rebrightening. Similar behavior of these bumps indicate that they might have the same origin. 

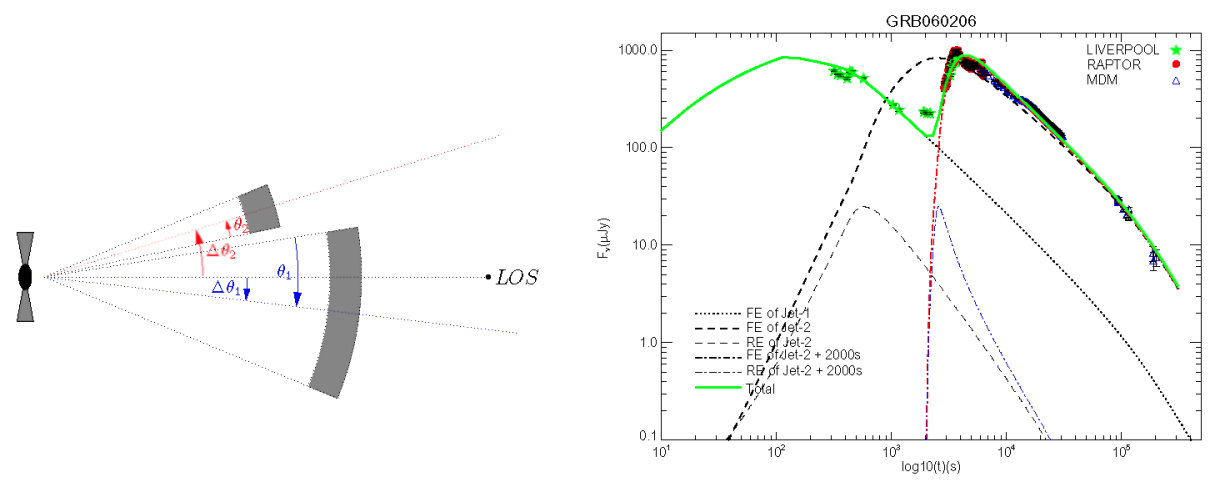

FIGURE 1. The left picture is the schematic two jets scenario for GRB060206 and the right is our fit to the R-band light curve of GRB 060206.

\section{MODEL}

The canonical X-ray afterglow behavior of a long lasting plateau in the light curve of early phase identified by Swift is regarded as the energy injection due to late time activities of the GRB central engine $[3,4,5]$. The origin of the central engine and its late activity are still open questions. It is generally accepted that the long bursts (duration $>2$ s) originate from collapsars $[6,7]$. On the other hand, short bursts (duration $<2 \mathrm{~s}$ ) are thought to be produced by the merging of compact objects (see [8], for a review). The formation of a hot and dense accretion disk is a common consequence of the above central engines. Reynoso et al. (2006) [9] argued that a rotating black hole can induce the surrounded neutrino-cooled accretion disk to precess and nutate. Their results imply that precession and nutation of those disks are possible in the context of GRB engines and the precession period varies between $0.01 \sim 10 \mathrm{~s}$ under typical GRB parameters. So, even when the jet produces the main burst is on-axis, it is possible that the collimated outflow supplied by the late activity of the central engine to departure from the lineof-sight (LOS) because of the precession of the torus of the central engine. Then the emission from the interaction between the off-LOS outflow and the ambient medium will contribute to the late afterglow.

We assume that the internal shocks produced within the on-axis jet (Jet 1) account for the main burst. The angle between the symmetry axis of Jet 1 and the LOS is $\Delta \theta_{1}$, as showed in Figure 1 on the left side. The half-opening angle of Jet 1 is $\theta_{1}$. After some time $\Delta t$, the central engine became active again and ejected another collimated off-axis jet (Jet 2) with half-opening angle $\theta_{2}$ and angle departure $\Delta \theta_{2}$ from the LOS. We use such two jets scenario to fit the afterglow of GRB 060206. 


\section{RESULT AND DISCUSSIONS}

The big re-brightening was due to the off-axis emission from the late second beamed jet including the zero time effect. The whole light curve is the combination of the emission coming from the interaction between the two jets and the ambient medium.

In our calculation, the forward-shock model for Jet 1 and the forward-reverse shock model for Jet 2 are applied. In Fig. 1, "FE" and "RE" represent the forward shock emission and the reverse shock emission, respectively in Figure 1. The fitting parameters for Jet 1 are: $\varepsilon_{e}=0.06, \varepsilon_{B}=0.0008, p=2.10, \theta_{1}=0.08, \Delta \theta_{1}=0.07, E_{\text {iso }}=5.8 \times 10^{52} \mathrm{erg}$, and for Jet 2 are: $\varepsilon_{e}=0.1, \varepsilon_{B}=0.01, p=2.12, \theta_{2}=0.04, \Delta \theta_{2}=0.05, E_{\text {iso }}=1.0 \times 10^{54}$ erg. The number density of the ambient medium is taken to be $n=50 \mathrm{~cm}^{-3}$. The dotted line represents the contribution from Jet 1 . The thick dashed line and thin dashed line correspond to the emissions from the forward shock and the reverse shock of Jet 2, respectively. The dash-dotted lines represent the emission from Jet 2 taking into account the zero-time effect $(\Delta t=2000 \mathrm{~s})$. The thick solid line includes the contribution from both Jet 1 and Jet 2. R-band data are taken from Monfardini et al. (2006), Stanek et al. (2007) and Woźniak et al. (2006) [10, 11, 12].

Our result indicates that only fine-tuned parameters can fit the observational data of GRB060206 well. A huge impulsive energy injection is predicted to account for the big re-brightening if the energy injection model is the true case. The collimation-corrected energy of the second jet in our scenario is even more larger, about 7 times that of the first jet. At least GRB 060206 is anomalous if our model works. As a conservative extrapolation, we suggest that GRB 970508 and GRB 060210, also displaying a remarkable re-brightening might also have a precessing torus/accretion.

A further consequence of our scenario is that the jet break is due to the off-axis second jet, not the jet produced the main burst. In this case, we can not estimate the isotropic energy of the second jet directly and must fit the afterglow data to get it. As a result, the procedure of deriving the jet opening angle from the break time obtained by fitting the light curve with a broken power-law function, as well as the isotropic energy, might be not directly applicable in many cases [11].

\section{REFERENCES}

1. D. Palmer, et a1., GCN Circ 4697 (2006).

2. J. P. U. Fynbo, et al., $A \& A$ 451, L47-L50 (2006).

3. J. A. Nousek, et al., $A p J$ 642, 389-400 (2006).

4. B. Zhang, et al., ApJ 642, 354-370 (2006).

5. P. T. O'Brien, et al., ApJ 647, 1213-1237 (2006).

6. S. E. Woosley, ApJ 405, 273-277 (1993).

7. A. I. MacFadyen, and S. E. Woosley, ApJ 524, 262-289 (1999).

8. E. Nakar, Physics Reports 442, 166-236 (2007).

9. M. M. Reynoso, G. E. Romero, and O. A. Sampayo, $A \& A 454,11-16$ (2006).

10. A. Monfardini, et al., ApJ 648, 1125-1131 (2006).

11. K. Z. Stanek, et a1., ApJ 654, L21-L24 (2007).

12. P.R.Woźniak, etal., ApJ 642, L99-L102 (2006). 